\title{
MATHEMATICAL MODELING, SIMULATION AND IDENTIFICATION OF MICRO COAXIAL HELICOPTER
}

\author{
Hari Muhammad, Huynh Phuoc Thien and Taufiq Mulyanto \\ Institut Teknologi Bandung \\ Faculty of Mechanical and Aerospace Engineering \\ Department of Aeronautics and Astronautics \\ Jl. Ganeca 10, Bandung 40132, Indonesia \\ tel.: +62 22 2504243, fax: +62 222534099 \\ e-mail:harmad@ae.itb.ac.id
}

\begin{abstract}
Micro Coaxial Helicopter with compact size and vertical takeoff ability offers a good Micro Aerial Vehicle (MAV) configuration to handle indoor mission such as search, rescue and surveillance. An autonomous MAV helicopter equipped with micro vision devices could provide more information of the scene, in which the human present is risky. Toward an autonomous flight, mathematical model of the helicopter should be obtained before controller design takes place. This paper will discuss the mathematical modelling, simulation and identification of a micro coaxial helicopter. The mathematical model of the micro coaxial helicopter will be presented, in which total forces and moment are expressed as a Taylor series expansion as function of the state and control variables. The mathematical model will be used to simulate the helicopter responses due to control input. The simulation was used to obtain better understanding of the characteristics of the helicopter before flight test program are performed. Flight test program dedicated to identify the parameter of the micro coaxial helicopter have been carried out. The micro coaxial helicopter was instrumented with sensory system to measure some input and output variables. The use of Kalman filter to estimate the state and total least squares to estimate the aerodynamic parameter of micro coaxial helicopter based on the flight test data will be presented. Some identification results and model validation will be given in this paper.
\end{abstract}

Keywords: micro coaxial helicopter, mathematical modelling, flight simulation, parameter identification

\section{Introduction}

Micro Aerial Vehicle (MAV) offers excellent tool to support missions in indoor environment. With certain onboard, intelligent the MAV can be used to perform tasks that may be dangerous for human or tasks in which human presence is not possible, e.g. search and rescue, surveillance in unstable contraction, in underground tunnel. In order to operate in indoor environments with limited space and rich of obstacles of different size and shape, the MAV should be inherently stable to simplify the control. Control system should be designed and implemented to autonomously control the MAV.

System identification approach is properly one of the good solutions to achieve an appropriate mathematical model. In detail, flight test data will be used to identify the parameters in the model. Unfortunately, due to the small size of the helicopter, and the use of low cost sensory system, data obtained from flight test are corrupted by noise. These inaccurate data could make the estimation of parameters become improper. In order to handle this issue, one of the solutions is using the Kalman filter followed by the total least square in the identification process.

The present paper starts with discussion of a mathematical model of a micro coaxial helicopter. Next, the numerical simulation prior to the flight test will be given to obtain proper values of control inputs during real flight test program. Parameter identification technique to estimate both the state and the aerodynamic parameters will be described. Estimation of the aerodynamic parameter based on real flight data will be presented and discussed. Some conclusions will be given in this paper. 


\section{Mathematical model of micro coaxial helicopter}

A typical micro coaxial helicopter is shown in Fig. 1. Two pairs of $50 \mathrm{~cm}$ diameter rotor blade are used to generate lift. These rotors are driven in opposite direction by two electric motors to counter the torque generated by rotor system [1].

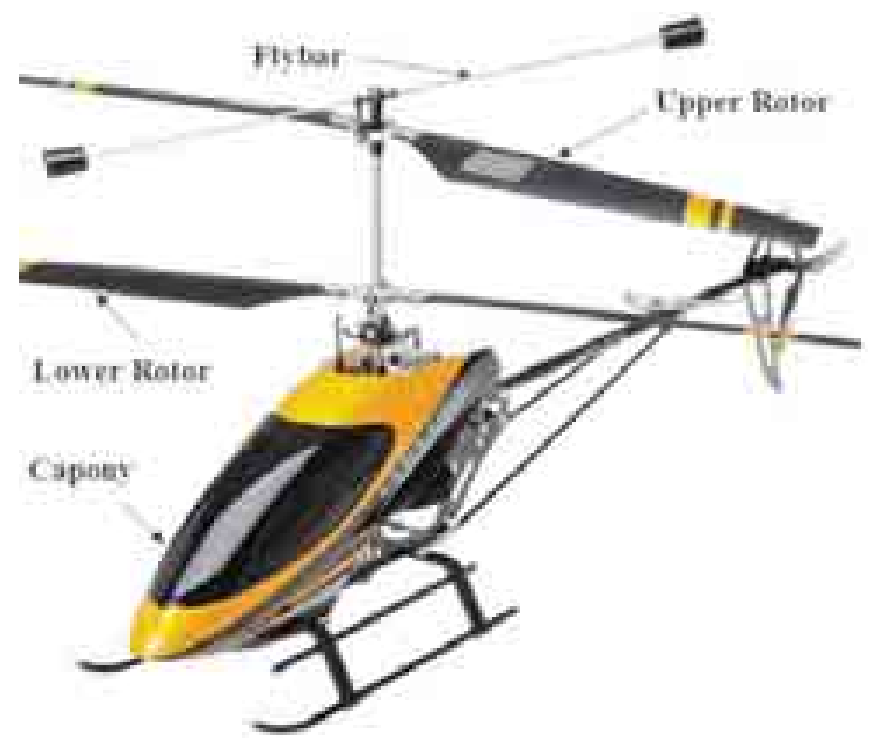

Fig. 1. Lama 400D coaxial helicopter [1]

In general, control of this type of rotorcraft is done by main motors and swash-plate system, both inactive and passive way. In upper rotor, a fly-bar is attached to the driven shaft. Through the mechanical linkages with rotor blade, this fly-bar will provide passive control into the upper rotor input. In lower rotor, swash-plate system with servomotors is used to control pitch and roll of the helicopter. To activate a motion in vertical direction, motor rotational speed should simultaneously be varied, while differential variation of these RPM will lead to a yaw motion.

\subsection{The longitudinal equations of motion}

The kinematics model of a Micro Coaxial Helicopter is a set of differential equations relating the forces and moments acting on the helicopter. This model can be derived from equation of motion of the helicopter. In the body-fixed reference frame, this equation can be written as follows, only the longitudinal equations are considered here [2].

$$
\begin{aligned}
& \dot{u}=-q w-g \sin \theta+\frac{X}{m}, \\
& \dot{w}=q u+g \cos \theta+\frac{Z}{m}, \\
& \dot{\theta}=q, \\
& \dot{q}=\frac{M}{I_{y}} \\
& \dot{h}=u \sin \theta-w \cos \theta .
\end{aligned}
$$

In equation (1), $u$ and $w$ indicate the velocity components of the helicopter along the $\mathrm{X}$ and $\mathrm{Z}$ axes respectively, $q$ is the pitch rate, $\theta$ denotes the angle of pitch, $h$ is the altitude, $g$ is the acceleration due to gravity, $\mathrm{m}$ is mass, and $\mathrm{I}_{\mathrm{y}}$ is the moment of inertia. 


\subsection{The rotor dynamic model}

The rotor dynamic model here is derived for the flapping angle of the lower rotor and the flybar, which are defined as follows [3, 4]:

$$
\begin{aligned}
& \dot{\alpha}_{\ell}=\tau_{\ell} \alpha_{\ell}+k_{\delta} \delta_{\text {lon }}-q, \\
& \dot{\alpha}_{f}=\tau_{f} \alpha_{f}-q .
\end{aligned}
$$

in which $\alpha_{\ell}$ and $\alpha_{f}$ are the flapping angle of the lower rotor and the fly-bar respectively, $\delta_{\text {lon }}$ denotes the deflection of the longitudinal control surface, and $q$ is the pitch rate. In equation (2), $\tau_{\ell}$, $\tau_{f}$, and $k_{\delta}$ are constants.

The upper rotor flapping angle $\alpha_{u}$ is modelled as a linear function of the flapping angle of the fly-bar as follows [2]:

$$
\alpha_{u}=k_{f} \alpha_{f}
$$

The flapping angle of both the upper and the lower rotors will be used to develop the aerodynamic-propulsion model of the micro coaxial helicopter.

\subsection{The aerodynamic-propulsion model}

The total aerodynamic-propulsion force along the longitudinal axis $X$, the total aerodynamicpropulsion force along the vertical axis $Z$, and the total aerodynamic-propulsion pitching moment in the lateral axis $M$, can be expressed as follows [5]:

$$
\begin{aligned}
& X=\frac{1}{2} \rho V^{2} S C_{X}, \\
& Z=\frac{1}{2} \rho V^{2} S C_{Z}, \\
& M=\frac{1}{2} \rho V^{2} S C_{m} R .
\end{aligned}
$$

where $C_{x}, C_{z}$, and $C_{m}$ denote the coefficients of the aerodynamic-propulsion forces along the $X$ and $Z$-axes, and pitching moment about $Y$-axis respectively, $V$ is the forward speed, $\rho$ is the air density.

By substituting $V=\omega R$ and $S=\pi R^{2}$ into equation (4), where $\omega$ and $R$ are the rotational speed and radius of the rotor respectively, it follows that:

$$
\begin{aligned}
& X=\frac{1}{2} \rho \pi \omega^{2} R^{4} C_{X}, \\
& Z=\frac{1}{2} \rho \pi \omega^{2} R^{4} C_{Z}, \\
& M=\frac{1}{2} \rho \pi \omega^{2} R^{5} C_{m} .
\end{aligned}
$$

The aerodynamic forces and moment coefficients can be expressed in terms of several state and control variables of the vehicle, for example the lower and upper flapping angles, pitch rate, deflection of the longitudinal control surface, and the thrust coefficient $T_{c}$ defined as

$$
T_{c}=\frac{T}{\frac{1}{2} \rho \pi \omega^{2} R^{4}} .
$$

The dependency of those coefficients on these variables can be expressed as follows: 


$$
\left\{C_{X} ; C_{Z} ; C_{m}\right\}=f\left(\alpha_{\ell}, \alpha_{u}, q, \delta_{l o n}, T_{c}\right) .
$$

Each coefficient in equation (7) can be expressed in a Taylor series expansion as a function of the state and control variables as well as thrust coefficient. If terms up to the first order are included, these coefficients can be expressed as follows $[6,7]$ :

$$
\begin{aligned}
& C_{X}=C_{X_{0}}+C_{X_{\alpha_{\ell}}} \alpha_{\ell}+C_{X_{\alpha_{u}}} \alpha_{u}+C_{X_{q}} q+C_{X_{\delta_{l o n}}} \delta_{\text {lon }}+C_{X_{T_{c}}} T_{c}, \\
& C_{Z}=C_{Z_{0}}+C_{Z_{\alpha_{\ell}}} \alpha_{\ell}+C_{Z_{\alpha_{u}}} \alpha_{u}+C_{Z_{q}} q+C_{Z_{\delta_{\text {lon }}}} \delta_{\text {lon }}+C_{Z_{T_{c}}} T_{c}, \\
& C_{m}=C_{m_{0}}+C_{m_{\alpha_{\ell}}} \alpha_{\ell}+C_{m_{\alpha_{u}}} \alpha_{u}+C_{m_{q}} q+C_{m_{\delta_{\text {lon }}}} \delta_{\text {lon }}+C_{m_{T_{c}}} T_{c} .
\end{aligned}
$$

In equation (8), $C_{X_{\alpha_{\ell}}}, C_{X_{\alpha_{u}}}, C_{X_{q}}, C_{X_{\delta_{l o n}}}, C_{Z_{\alpha_{\ell}}}, \ldots C_{m_{\alpha_{\ell}}}, C_{m_{\delta_{l o n}}}$ are the aerodynamic and control parameters, and $C_{x_{T_{c}}}, C_{z_{T_{c}}}$, and $C_{m_{c}}$ are the thrust parameters expressing the effect of the thrust on the total aerodynamic forces and moment. These parameters can be determined from both theoretical prediction or experimental. In this paper, these parameters will be estimated from flight test experiment.

Substitution of equation (8) into (4) and the result is substituted into (1) yields the differential equation of motion of the micro coaxial helicopter as follows.

$$
\begin{aligned}
& \dot{u}=-q w-g \sin \theta+\frac{1}{2} \frac{\rho \omega^{2} R^{4}}{m}\left(C_{X_{0}}+C_{X_{\alpha_{\ell}}} \alpha_{\ell}+C_{X_{\alpha_{u}}} \alpha_{u}+C_{X_{q}} q+C_{X_{\delta_{l o n}}} \delta_{l o n}+C_{X_{T_{c}}} T_{c}\right), \\
& \dot{w}=q u+g \cos \theta+\frac{1}{2} \frac{\rho \omega^{2} R^{4}}{m}\left(C_{Z_{0}}+C_{Z_{\alpha_{\ell}}} \alpha_{\ell}+C_{Z_{\alpha_{u}}} \alpha_{u}+C_{Z_{q}} q+C_{Z_{\delta_{l o n}}} \delta_{l o n}+C_{Z_{T_{c}}} T_{c}\right), \\
& \dot{\theta}=q, \\
& \dot{q}=\frac{1}{2} \frac{\rho \omega^{2} R^{5}}{I_{y}}\left(C_{m_{0}}+C_{m_{\alpha_{\ell}}} \alpha_{\ell}+C_{m_{\alpha_{u}}} \alpha_{u}+C_{m_{q}} q+C_{m_{\delta_{l o n}}} \delta_{l o n}+C_{m_{T_{c}}} T_{c}\right), \\
& \dot{h}=u \sin \theta-w \cos \theta .
\end{aligned}
$$

In principle, the differential equations (2) and (9) can be used to simulate the flight test scenarios as will be explained in the following section.

\section{Flight simulation of micro coaxial helicopter}

The purpose of the flight simulation is to test the mathematical model of the helicopter as well as to obtain the reference values of control input for later use in flight test program. The mathematical model of the helicopter given in equations (2) and (9) is implemented into the MATLAB/Simulink software. Fig. 2 shows the schematic diagram of the implementation of the equations of motion of the micro coaxial helicopter [2].

The physical parameters of the micro coaxial helicopter used in the simulation are presented in Tab. 1, see also Fig. 1.

Tab. 1. Micro coaxial helicopter data

\begin{tabular}{|l|l|}
\hline Characteristics & Data \\
\hline Main rotor radius & $25 \mathrm{~cm}$ \\
\hline Total weight & $800 \mathrm{~g}$ \\
\hline Engine type & Electric motor \\
\hline Distance between rotor & $80 \mathrm{~mm}$ \\
\hline Endurance & 10 minutes \\
\hline
\end{tabular}




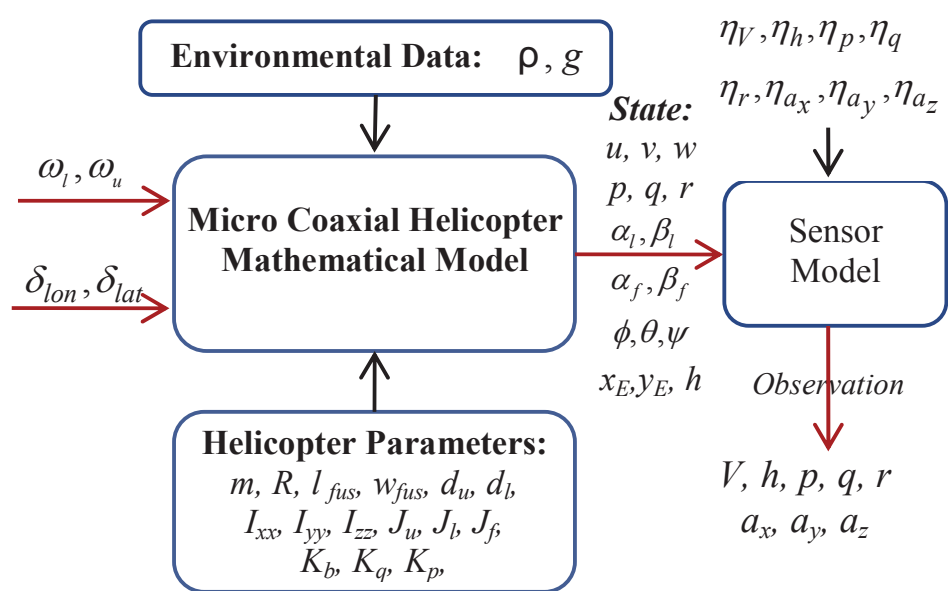

Fig. 2. Schematic diagram of the implementation of the mathematical model of micro coaxial helicopter

The simulation was carried out within 20 seconds and time step of $0.01 \mathrm{~s}$. The total forces $X$ and $Z$ as well as the total moment $M$ in equation (4) are assumed known [2]. The time history of the control input for the rotor and the servo system in longitudinal simulation are shown in Fig. 3. The corresponding response in term of translational velocity $(u, w)$, angular rate about $Y$-axis (q), pitch angle $(\theta)$ and altitude $(h)$ are depicted in Fig. 4.
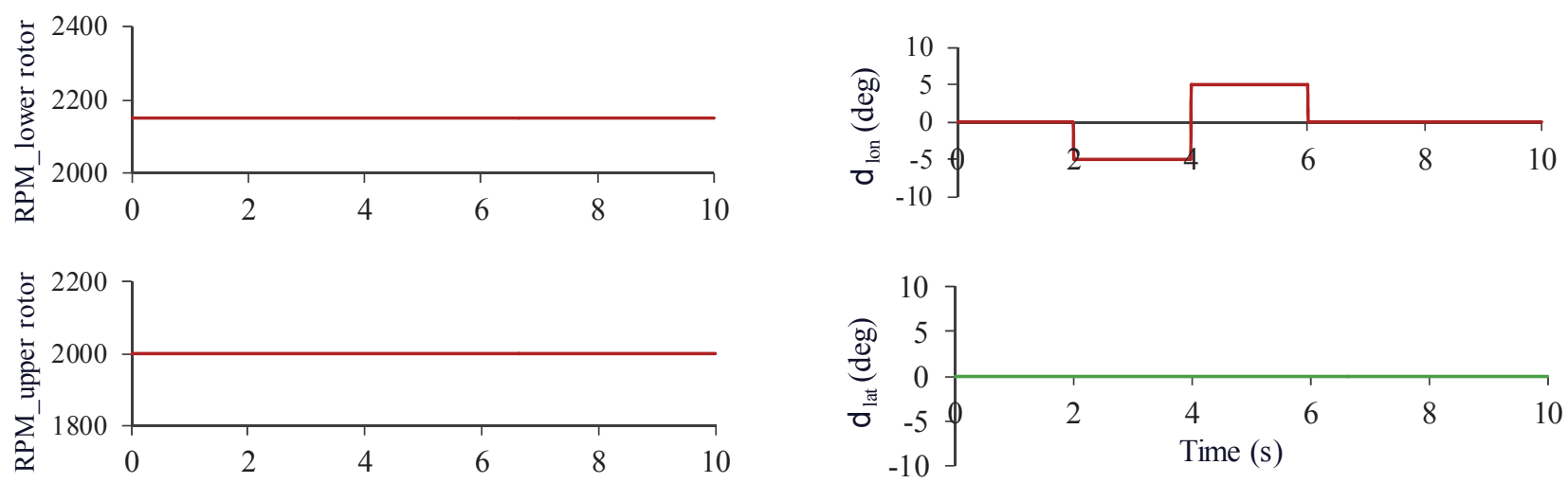

Fig. 3. Control input of rotor and servo system in longitudinal maneuver
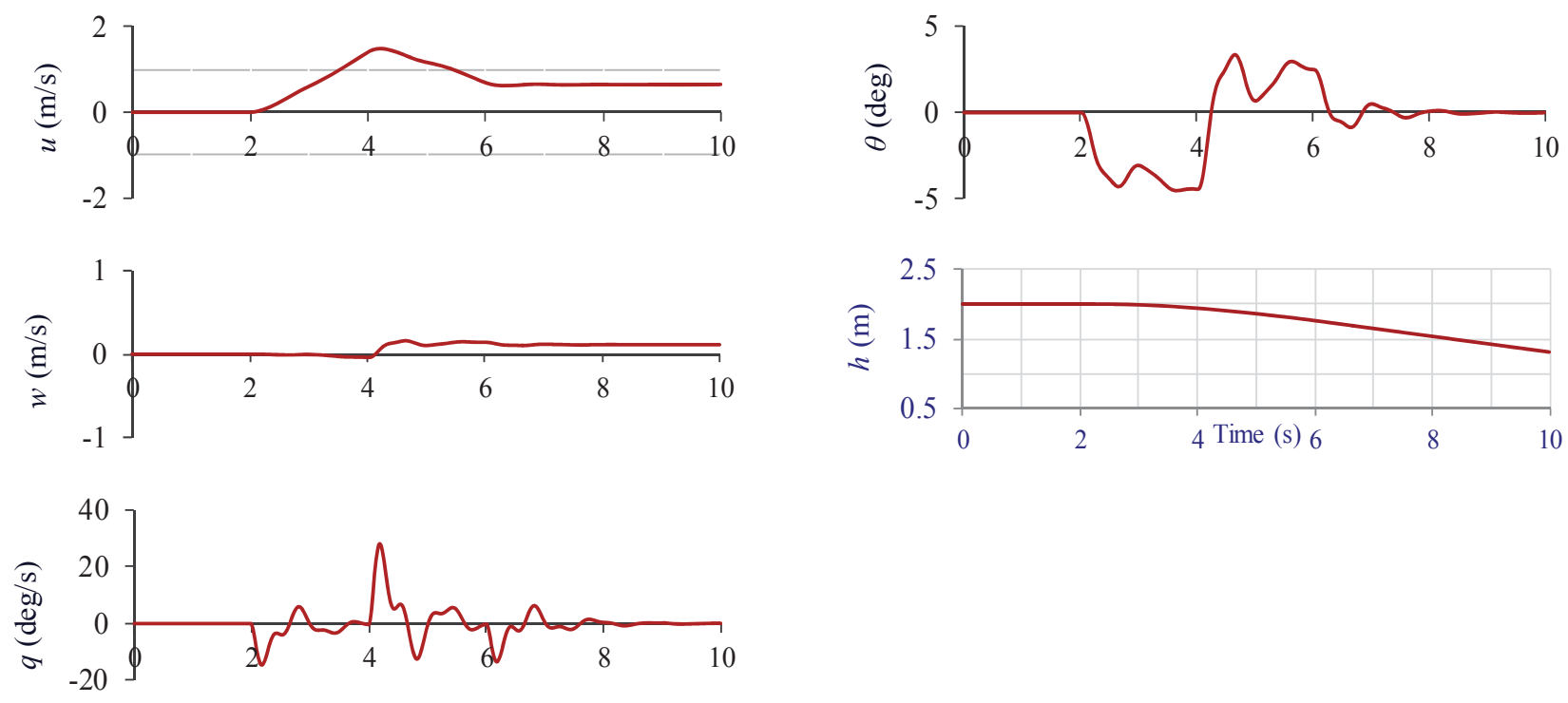

Fig. 4. Response of the helicopter to the control input in term of translational velocity (u, w), angular rate about $Y$ axis $(q)$, pitch angle $(\theta)$, and altitude $(h)$ 
Figure 5 shows the observed speed $\left(V_{m}\right)$ and altitude $\left(h_{m}\right)$. Also presented in Fig. 5 are the accelerations along the longitudinal and vertical axes $\left(a_{x}, a_{z}\right)$, pitch rate $(q)$, and upper and lower flapping angles $\left(\alpha_{u}, \alpha_{l}\right)$. In this simulated flight data, it is assumed that noise was presence in the observed data.
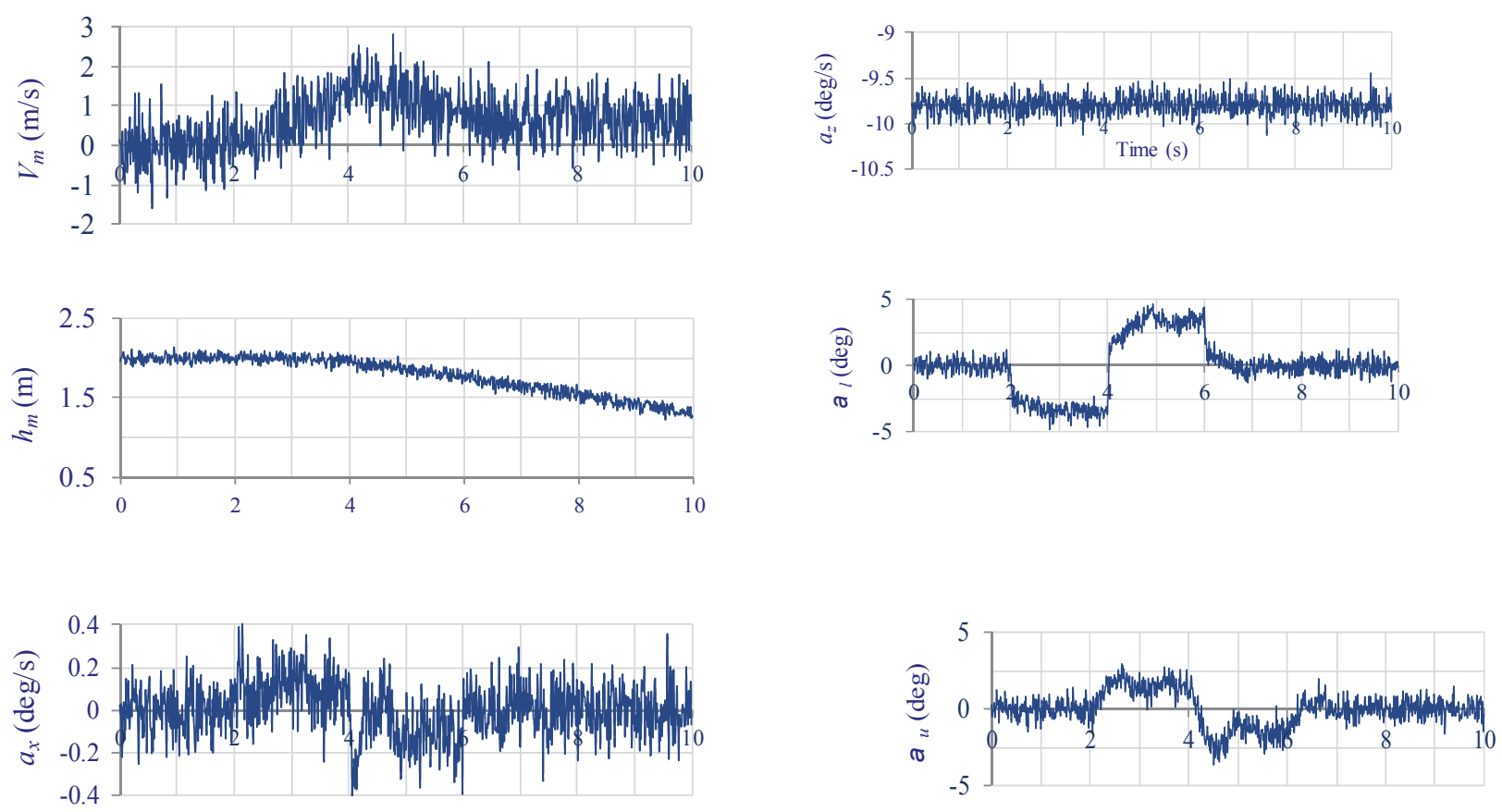

Fig. 5. The observed response of the helicopter due to control input: airspeed, altitude, vertical acceleration, rate of pitch, lower, and upper flapping angles. Noise is added in these observed responses

The simulated flight data as given in Fig. 3 and Fig. 5 can be used to obtain proper values of control inputs during real flight test program $[2,7]$. Also, the simulated flight data was used to validate the software of the Kalman filter and the total least squares [6,9].

\section{Identification of micro coaxial helicopter parameters}

In the identification of micro coaxial helicopter parameters, two steps parameter estimation are used. In the first step, Extended Kalman Filter was used to estimate the states of the helicopter. The Total Least Square was then applied to estimate the parameters of the aerodynamic force and moment models.

\subsection{The flight instrumentation system and data}

Flight test program aimed to identify the aerodynamic-propulsion parameter was carried out using modified micro coaxial helicopter. The helicopter was instrumented with three navigation sensors:

- 6 DOF Inertial Measurement Unit (IMU) from SparKFun Electronic to provide the measurement of the airframe accelerations $\left(a_{x}, a_{y}, a_{z}\right)$, measured range of $6 \mathrm{~g}$, and angular rates $(p, q, r)$, measured range of $500 \mathrm{deg} / \mathrm{s}$; Data rate of this IMU is up to $200 \mathrm{~Hz}$,

- Digital magnetic compass (DMC) with tilt compensated OS500-S from Ocean Server for sensing heading attitude, tilt angle in longitudinal and lateral direction (resolution: $0.1^{0}$, data rate: $40 \mathrm{~Hz}$ ),

- Sonar Range Finder sensor for measuring the helicopter altitude (range: $15 \mathrm{~cm}-650 \mathrm{~cm}$ ). 
Also, two optical shaft encoders are used to record the rotational speed of rotor system. Two microcontrollers are selected to read the servo's control input, to collect, and to encode the entire sensor reading into data packages. The architecture of the sensory system is depicted in Fig. 1.

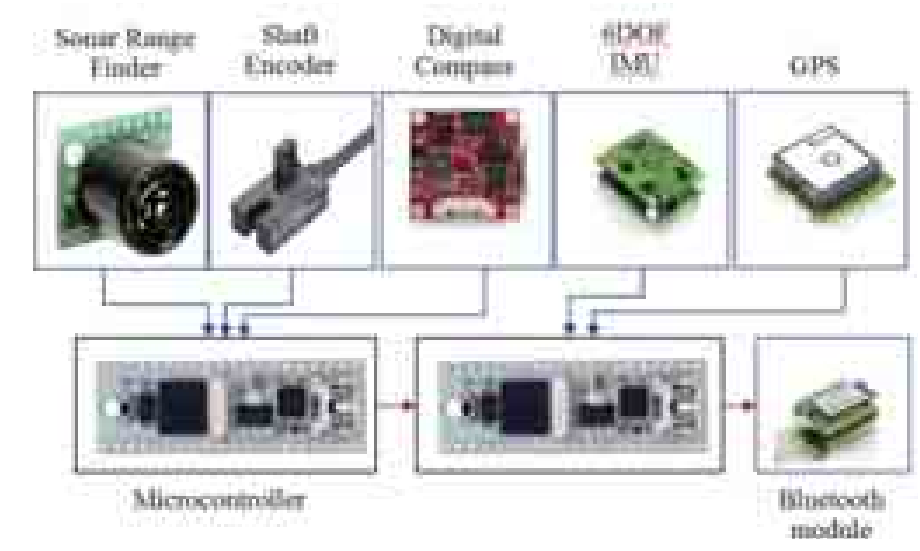

Fig. 1. Schematic diagram of overall structure of flight test instrumentation system

Fig. 2 shows typical example of input-output flight test data obtained in longitudinal maneuver. In this figure, the time history of control inputs is shown in the left side; while the helicopter responses are shown in the right side. It can be seen that the measured data obtained from the sensory system are noisy with high noise to signal ratio. Therefore, the filtering of data was carried out to obtain appropriate data for further processing [7].
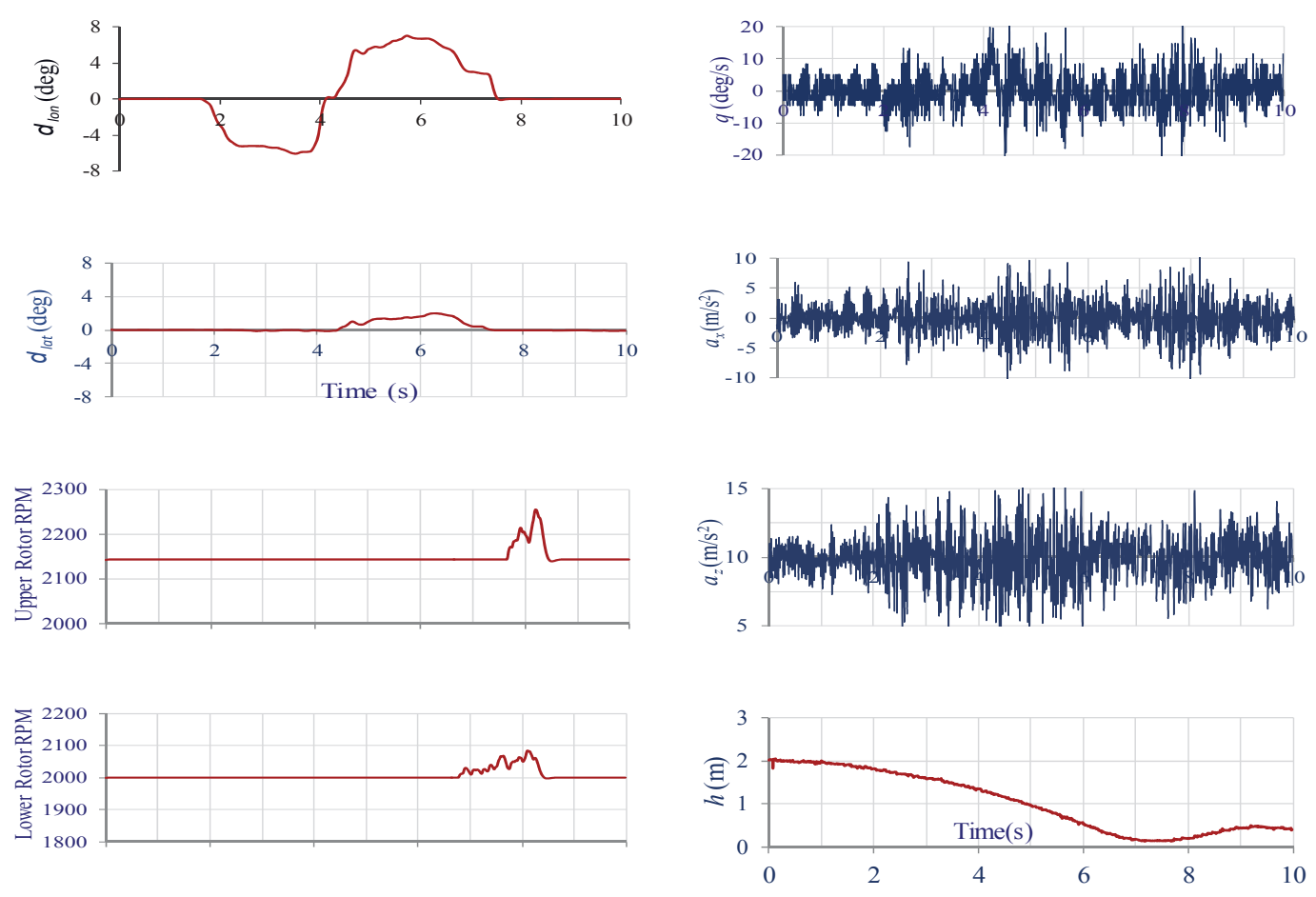

Fig. 2. Control input and measured data obtained from longitudinal flight test maneuver.

\subsection{State estimation}

Assuming that the measurement of accelerations and angular rates of the micro coaxial helicopter can be performed using IMU with sufficient accuracy. By replacing the total forces in 
equation (1) with the specific forces $a_{x_{m}}=\frac{X}{m} ; a_{z_{m}}=\frac{Z}{m}$, the equations of longitudinal motion of the micro coaxial helicopter can be expressed as follows [8]:

$$
\begin{aligned}
& \dot{u}=-q w-g \sin \theta+a_{x_{m}}, \\
& \dot{w}=q u+g \cos \theta+a_{z_{m}}-\lambda_{z}, \\
& \dot{\theta}=q_{m}, \\
& \dot{h}=u \sin \theta-w \cos \theta, \\
& \dot{\lambda}_{z}=0 .
\end{aligned}
$$

In equation (10), the bias in the vertical acceleration is considered and it is assumed constant so that the derivative with respect to time is zero. Also, the subscript $m$ in equation (10) denotes the measured variable.

In principle, Extended Kalman Filter (EKF) can be applied to estimate the state in . (10) as the input in term of translational acceleration $a_{x_{m}}, a_{z_{m}}, q_{m}$ are known from the flight test. In addition, the flapping angle of the upper and lower rotor can be obtained by integrating equation with $\delta_{\text {lon }}$ and $q_{m}$ acquired in the flight test and using equation (3).

Results on the estimation of translational velocity $(\mathrm{u}, \mathrm{v})$, pitch angle $(\theta)$ bias in vertical acceleration and altitude in longitudinal maneuver are shown in Fig. 3. The estimated flapping angle of the upper and lower rotor in longitudinal maneuver is depicted in Fig. 4.
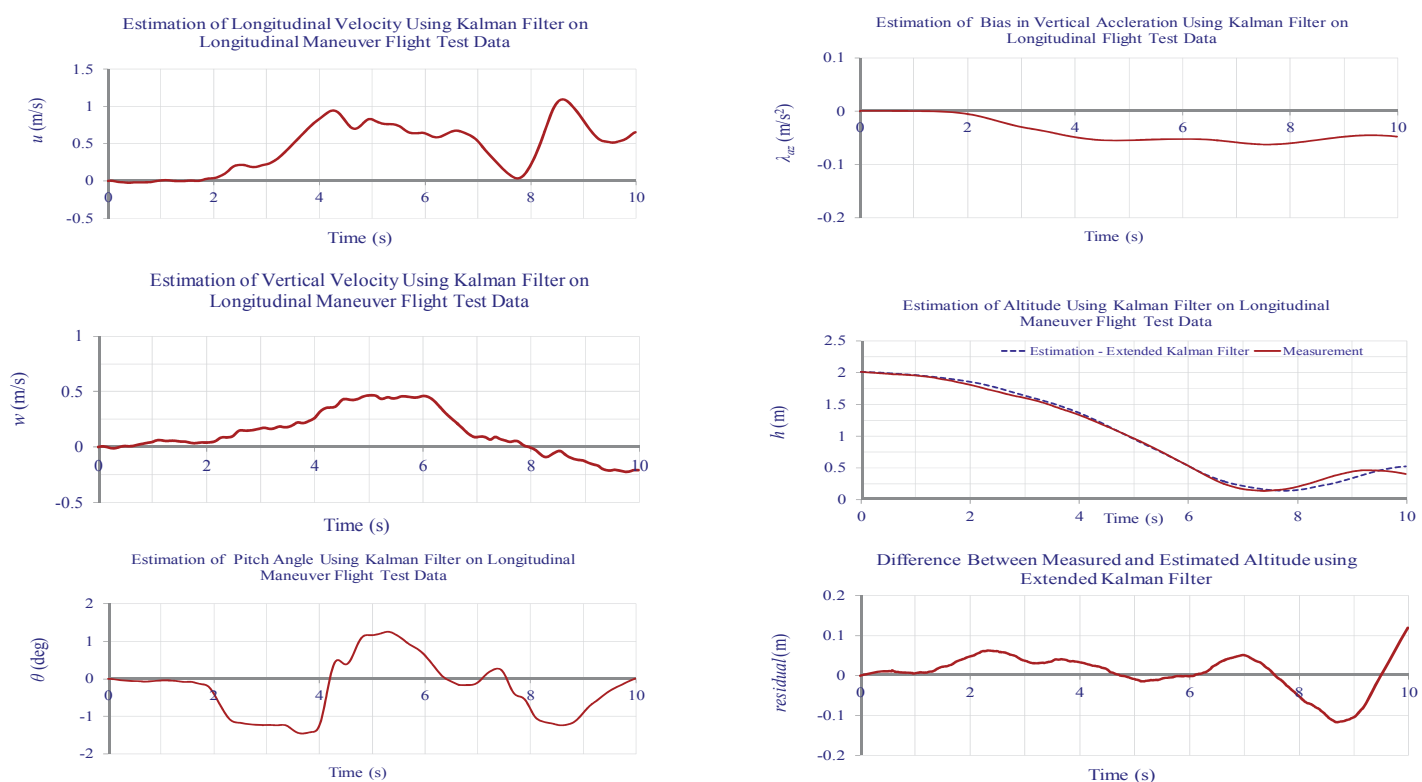

Fig. 3. Estimated of translational velocity $(u, v)$, pitch angle $(\theta)$ bias in vertical acceleration and altitude


Fig. 4. Estimated of the flapping angle of the lower and upper rotor in longitudinal maneuver 


\subsection{Parameter estimation}

The aerodynamic-propulsion model given in equations (5) and (8) can be rewritten as:

$$
\begin{aligned}
& C_{X}=\frac{X}{\frac{1}{2} \rho \pi \omega^{2} R^{4}}=\frac{m a_{x_{m}}}{\frac{1}{2} \rho \pi \omega^{2} R^{4}}=C_{X_{0}}+C_{X_{\alpha_{\ell}}} \alpha_{\ell}+C_{X_{\alpha_{u}}} \alpha_{u}+C_{X_{q}} q+C_{X_{\delta_{l o n}}} \delta_{\text {lon }}+C_{X_{T_{c}}} T_{c}, \\
& C_{Z}=\frac{Z}{\frac{1}{2} \rho \pi \omega^{2} R^{4}}=\frac{m a_{z_{m}}}{\frac{1}{2} \rho \pi \omega^{2} R^{4}}=C_{Z_{0}}+C_{Z_{\alpha_{\ell}}} \alpha_{\ell}+C_{Z_{\alpha_{u}}} \alpha_{u}+C_{Z_{q}} q+C_{Z_{\delta_{\text {lon }}}} \delta_{\text {lon }}+C_{Z_{T_{c}}} T_{c}, \\
& C_{m}=\frac{M}{\frac{1}{2} \rho \pi \omega^{2} R^{5}}=\frac{I_{y} \dot{q}_{m}}{\frac{1}{2} \rho \pi \omega^{2} R^{5}}=C_{m_{0}}+C_{m_{\alpha_{\ell}}} \alpha_{\ell}+C_{m_{\alpha_{u}}} \alpha_{u}+C_{m_{q}} q+C_{m_{\delta_{l o n}}} \delta_{\text {lon }}+C_{m_{T_{c}}} T_{c} .
\end{aligned}
$$

The force coefficients $C_{X}$ and $C_{Z}$ in equation (11) can be obtained from measurement of longitudinal and vertical accelerations. The moment coefficient $C_{m}$ is obtained from numerical differentiation of $q_{m}$ by taking the 4 points Lagrange time derivative [8].

From the flight test data, the value of angular rate $q$, the control input of pitch servo $\delta_{\text {lon }}$ and the rotor system thrust coefficient $T_{c}$ are obtained. The flapping angles of the upper and lower rotor can be reconstructed as discussed in 0 . The principle of the regression method can be explained in short as follows. The aerodynamic model in equation (11) can be expressed as follows:

$$
y(i)=\theta_{0}+\theta_{1} x_{1}(i)+\theta_{2} x_{2}(i)+\ldots+\theta_{p} x_{p}(i)+\varepsilon(i),
$$

where $y(i)$ is the dependent variable, i.e. the aerodynamic force and moment coefficients, $x_{p}(i)$ denote the independent variables, i.e. the (estimated) state and control variables, $\theta_{p}$ is the vector of the aerodynamic parameters, and $\varepsilon(i)$ am the stochastic equation error, accounting for measurement as well as model errors on the dependent variable. In the compact matrix form, equation (12) can be written as follows:

$$
y=X \theta+\varepsilon .
$$

The matrix of independent variables $X$ is assumed known exactly from the state estimation, i.e. using EKF, or from direct measurement with highest accuracy of the instrumentation system. Using the Least Squares (LS) method [10], the parameter vector $\theta$ can be estimated from:

$$
\theta_{L S}=\left[X^{T} X\right]^{-1}\left[X^{T} X\right] y \text {. }
$$

However, due to the limited accuracy of the instrumentation system in the measurement or reconstruction of the flapping angle or the upper and the lower: $\alpha_{u}$ and $\alpha_{l}$, pitch rate $q$, deflection of the longitudinal control surface $\delta_{\text {lon, }}$ and thrust coefficient $T_{c}$, the data matrix $X$ has also error. Therefore, equation (13) can be written as follows:

$$
y=[X+\Delta X] \theta+\varepsilon .
$$

In equation (15), not only the observation $y$ has error but also the data matrix $X$ has error as well. Again, with the assumption that the dependent variable y can be measured with finite accuracy, equation (15) can be written as follows:

$$
y=[X+\Delta X \mid \theta] .
$$

Denote $C=[X y]$ is a compound matrix that contains both observation $y$ and data matrix $X$ in equation (16). This matrix $C$ could be decomposed into:

$$
C=U \Sigma V^{T}
$$


where $\Sigma=\operatorname{diag}\left(\sigma_{1}, \sigma_{2}, \ldots \sigma_{n+d}\right)$ is a single value decomposition of $C, \sigma_{1}>\sigma_{2}>\ldots>\sigma_{n+d}$ be the singular values of $C, n$ and $d$ is the number of independent variables and number of observation respectively. The element matrix in (17) could be partitioned based on $n$ and $d$ as follows

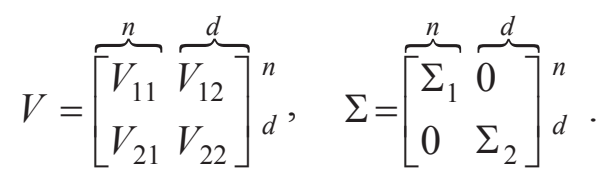

Solution of total least square exists if and only if $V_{22}$ is non-singularity. This solution is unique if and only if $\sigma_{n} \neq \sigma_{n+1}$. The existed solution could be given by

$$
\theta_{T L S}=-V_{12} V_{22}^{-1} \text {. }
$$

TLS was chosen as the estimator due to its ability in giving consistent estimation of parameters in the presence of noise in the measured data [9]. Tab. 2 presents the results on estimation of parameters in the longitudinal force and moment coefficient by applying the Total Least Squares on the flight test data.

Tab. 2. Estimated parameters in aerodynamic-propulsion model of micro coaxial helicopter

\begin{tabular}{cccccc}
\hline Parameters & Value & Standard Deviation & Parameters & Value & Standard Deviation \\
\hline$C_{X_{0}}$ & 0.0134 & 0.0042 & $C_{Z_{\delta_{l o n}}}$ & -0.1802 & 0.0108 \\
$C_{X_{\alpha_{u}}}$ & -0.0655 & 0.0149 & $C_{Z_{T_{l}}}$ & 0.0004 & 0.0009 \\
$C_{X_{\alpha_{l}}}$ & 0.3900 & 0.0218 & $C_{Z_{T_{u}}}$ & -0.0057 & 0.0007 \\
$C_{X_{q}}$ & 0.0569 & 0.0039 & $C_{m_{0}}$ & 0.0189 & 0.0068 \\
$C_{X_{\delta_{l o n}}}$ & -0.3569 & 0.0201 & $C_{m_{\alpha_{u}}}$ & 0.2370 & 0.0241 \\
$C_{X_{T_{l}}}$ & 0.0041 & 0.0016 & $C_{m_{\alpha_{l}}}$ & -0.6448 & 0.0354 \\
$C_{X_{T_{u}}}$ & -0.0063 & 0.0012 & $C_{m_{q}}$ & -0.0833 & 0.0063 \\
$C_{Z_{0}}$ & 0.0495 & 0.0022 & $C_{m_{\delta_{L o n}}}$ & 0.5995 & 0.0326 \\
$C_{Z_{\alpha_{u}}}$ & 0.0124 & 0.008 & $C_{m_{T_{l}}}$ & -0.0066 & 0.0026 \\
$C_{Z_{\alpha_{l}}}$ & 0.2034 & 0.0117 & $C_{m_{T_{u}}}$ & 0.0009 & 0.002 \\
$C_{Z_{q}}$ & 0.0285 & 0.0021 & & & \\
\hline
\end{tabular}

The comparison between the estimation of $C_{X}, C_{Z} C_{m}$ and the corresponding measured values are shown in Fig. 5. In general, the estimated values of $C_{X}, C_{Z}$, and $C_{m}$ fit well the measured values, except some periods in which transition of control input were made, i.e. at $t=2, t=4, t=8 \mathrm{~s}$, see also $\delta_{\text {lon }}$ in Fig. 2.

To evaluate the quality of the estimation of force and moment coefficients using Total Least Squares, the goodness of fit coefficient is used. This coefficient is defined as the correlation coefficient as follows:

$$
R^{2}=\frac{\left[y_{m}-y_{e}\right]^{T}\left[y_{m}-y_{e}\right]}{\left[y_{e}-\bar{y}\right]^{T}\left[y_{e}-\bar{y}\right]},
$$

in which $y_{m}$ and $y_{e}$ are the measured and estimated values of $C_{X}, C_{Z}$ and $C_{m}$ respectively, and $\bar{y}$ is the mean value of $y_{e}$. The correlation coefficients of $C_{X}, C_{Z}$ and $C_{m}$ obtained from analysis of the flight test data as presented in Fig. 10. are 0.91, 0.92 and 0.97, receptively. 

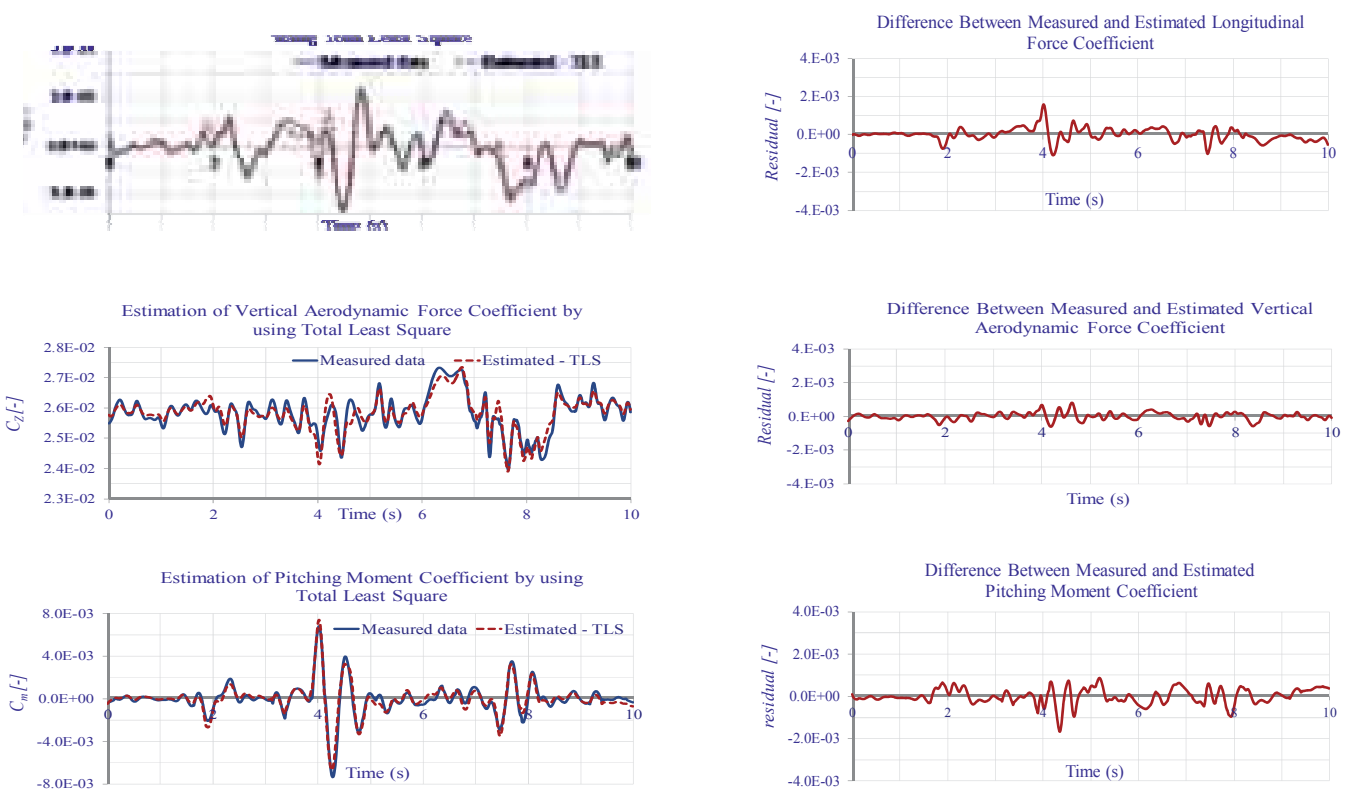

Fig. 5. Comparison between estimated and measured valued of $C_{X}, C_{Z}, C_{m}$ and the corresponding difference

\subsection{Model validation}

To validate the identified mathematical model of the micro coaxial on the longitudinal, the linearized aerodynamic force and moment model obtained from the system identification process will be substituted into the mathematical model of the helicopter. Simulation will be carried out on this model. The obtained results will be compared with simulation results of the analytical model to evaluate the quality of the identified model. The validation results are shown in Fig. 6.


Fig. 6. Validation of the estimation of the helicopter states: airspeed, angular velocity, flapping angle of the lower rotor and flybar, and the position in the longitudinal and vertical and axis

In general, the estimation results using the longitudinal mathematical model with linearized aerodynamic force and moment coefficient match the simulation results from analytical model rather well. These matching indicate that the identified model can capture well the dynamics of the 
helicopter. It is a good starting point for further improvement towards the obtaining of high accuracy mathematical model of the coaxial helicopter.

\section{Conclusion}

The mathematical model of micro coaxial helicopter has been derived in this paper. The model was used to simulate the helicopter. Also, the mathematical model has been used to identify the parameter from the real flight test data. The mathematical model of the micro coaxial helicopter derived from the identification result showed in good agreement with mathematical model obtained from the analytical approach.

In the future work, more flight tests should be performed to validate the identified dynamic model, repeating flight test run (execution) in certain number is necessary to ensure the consistent result of the parameters. Mathematical model of the micro coaxial helicopter in other mode, i.e. lateral, vertical maneuver should also be developed to obtain the full mathematical model of this kind of micro coaxial helicopter.

\section{References}

[1] www.helipal.com/walkera-hm-lama-400d-helicopter-yellow-2-4g-metal-edition.html, last visited: January 26, 2012.

[2] Thien, H. P., System modelling and identification of coaxial rotor micro aerial vehicle, Ph.D. Dissertation, Department of Aeronautics and Astronautics, Institut Teknologi Bandung, Bandung, Indonesia 2012.

[3] Schafroth, D., Boaubdallah, S., Bermes, C., Siegwart, R., Modelling and system identification of muFly micro helicopter, Journal of Intelligent Robot System, pp. 27-47, 2010.

[4] Shin, J., Fujiwara, D., Nonami, K., Hazawa, K., Model-based optimal attitude and positioning control of small-scale unmanned helicopter, Journal of Robotica, Vol. 23, pp. 51-63, 2005.

[5] Leishman, J. G., Principle of Helicopter Aerodynamics. Cambridge University Press, pp. 134$138,2000$.

[6] Muhammad, H., Thien, H. P., Mulyanto, T., Estimation of aerodynamic parameter of micro aerial vehicle using total least squares, Proceedings of the $4^{\text {th }}$ Regional Conference on Mechanical and Aerospace Technology, pp. 441-447, Ho Chi Minh City, Vietnam 2012.

[7] Thien, H. P., Mulyanto, T., Muhammad, H., Suzuki, S., Mathematical modelling and experimental system identification of micro coaxial helicopter dynamics, International Journal of Basic \& Applied Science IJBAS-JENS, Vol. 12, No. 02, pp. 88-102, April 2012.

[8] Thien, H. P., Mulyanto, T., Muhammad, H., Modelling and identification of longitudinal dynamic model of micro coaxial helicopter, Proceedings of the $4^{\text {th }}$ Regional Conference on Mechanical and Aerospace Technology, pp. 468-475, Ho Chi Minh City, Vietnam 2012.

[9] Muhammad, H., Thien, H. P., Mulyanto, T., Total least squares estimation of aerodynamic parameter of micro coaxial helicopter from flight data, International Journal of Basic \& Applied Science IJBAS-IJENS, Vol. 12, No. 02, pp. 44-52, April 2012.

[10] Mettler, B., Identification modelling and characteristics of miniature rotorcraft, Kluwer Academic Publishers, Boston 2003.

[11] Laban, M., Masui, K., Total least square estimation of aerodynamics model parameters from flight data, Journal of Aircraft, Vol. 30, No. 1, pp. 150-152, 1992. 